\title{
An Algorithm for Estimation of Flow Length Distributions Using Heavy-Tailed Feature
}

\author{
Weijiang Liu, Jian Gong, Wei Ding, and Guang Cheng \\ Department of Computer Science and Engineering, \\ Southeast University, 210096 Nanjing, Jiangsu, China \\ \{wjliu, jgong, wding, gcheng\}@njnet.edu.cn
}

\begin{abstract}
Routers have the ability to output statistics about packets and flows of packets that traverse them. Since however the generation of detailed traffic statistics does not scale well with link speed, increasingly passive traffic measurement employs sampling at the packet level. Packet sampling has become an attractive and scalable means to measure flow data on high-speed links. However, knowing the number and length of the original flows is necessary for some applications. This paper provides an algorithm that uses flow statistics formed from sampled packet stream to infer the absolute frequencies of lengths of flows in the unsampled stream. We achieve this through statistical inference and by exploiting heavy-tailed feather. We also investigate the impact on our results of different packet sampling rate. The experiment results show the inferred distributions are accurate in most cases.
\end{abstract}

\section{Introduction}

With the rapid increase of network link speed, packet sampling has become an attractive and scalable means to measure flow data. However, knowing the number and lengths of the unsampled flows remains useful for characterizing traffic and the resources required to accommodate its demands. Here are some applications: Resources Required for Collecting Flow Statistics: flow cache utilization and the bandwidth for processing and transmitting flow statistics are sensitive to the sampling rate, the number of flows, and flow lengths and duration; see $[1,2]$. Characterizing Source Traffic: the measured numbers of flows and the distribution of their lengths have been used to evaluate gains in deployment of web proxies [3], and to determine thresholds for setting up connections in flow-switched networks [4]. Sampling entails an inherent loss of information. We expect use statistic inference to recover information as much as possible. However, more detailed characteristics of the original traffic are not so easily estimated. Quantities of interest include the number of packets in the flow-we shall refer to this as the flow length-and the number of flows with fixed length.

\section{$1.1 \quad$ Related Work}

Kumar et al proposed a novel SCBF that performs per-flow counting without maintaining per-flow state in [5] and an algorithm for estimation of flow size 
distribution in [6]. Its disadvantage is that all packet must be processed due to not using sampling. Hohn and Veitch in [7] discussed the inaccuracy of estimating flow distribution from sampled traffic, when the sampling is performed at the packet level.

Although sampled traffic statistics are increasingly being used for network measurements, to our knowledge few studies have addressed the problem of estimating flow size distribution from the sampled packet stream. In [2], the authors studied the statistical properties of packet-level sampling using realworld Internet traffic traces. This is followed by [8] in which the flow distribution is inferred from the sampled statistics. After showing that the naive scaling of the flow distribution estimated from the sampled traffic is in general not accurate, the authors propose an EM algorithm to iteratively compute a more accurate estimation. Scaling method is simple, but it exploits the sampling properties of SYN flows to estimate TCP flow frequencies; EM algorithm does not rely on the properties of SYN flows and hence is not restricted to TCP traffic, but its versatility comes at the cost of computational complexity.

\subsection{Some Elementary Concepts}

This paper considers sampling some target proportion $p=1 / N$ of the packet stream. There are a number of different ways to implement this. Implementations include independent sampling of packets with probability $p=1 / N$, and periodic selection of every $N^{t h}$ packet from the full packet stream. In both cases we will call $N$ the sampling period, i.e., the reciprocal of the average sampling rate. Although the length distributions by random and periodic sampling can be distinguished, the differences are, in fact, sufficiently small [8]. A flow is defined as a stream of packets subject to flow specification and timeout. When a packet arrives, the specific rules of flow specification determine which active flow this packet belongs to, or if no active flow is found that matches the description of this packet, a new flow is created. A TCP flow is a stream of TCP packets subject to timeout and having the same source and destination IP addresses, same source and destination port numbers. Similarly, a UDP flow is a stream of UDP packets associated with above specification. A general flow is a stream of packets subject to timeout and having the same source and destination IP addresses, same source and destination port numbers(not considering protocol). In this paper, we will use the term original flow to describe the above flow. A sampled flow is defined as a stream of packets that are sampled at probability $p=1 / N$ from an original flow.

\subsection{Contribution and Outline}

This paper presents a novel algorithm for estimation of flow size distributions from sampled flow statistics. Our method is available not only to TCP flows but also to general flows. We complete this work using four approaches. The first formalizes the probability distribution of original flow length of a sampled flow length $j$. The second classifies two types of flows based on their probability that 
no packet is sampled. A flow is labeled as small (S) when it's probability that no packet is sampled is more than $\varepsilon$ and as large (L) when it's probability that no packet is sampled is less than or equal to $\varepsilon$. The third gives a simple estimation method for large flows. The fourth uses maximum likelihood estimation and EM algorithm to estimate the full distribution of small flows.

The rest of this paper is organized as follows. In Section 2 we analyze the probability models of the original flow length distributions of a sampled flow under the assumptions of Pareto distributions. In Section 3, we classify two types of flows: small flow and large flow. Then we present different estimation methods for small flows and large flows, respectively. In Section 4 we discuss the computational complexity of our method. Furthermore, we compare our method with EM algorithm in estimation accuracy and computational complexity. We conclude in Section 5.

\section{Probability Distribution of Original Flow Length}

For a specific original flow $F$, let $X_{F}$ denote the number of packets in $F$, $Y_{F}$ denote the number of packets in the sampled flow from $F$. The conditional distribution of $Y_{F}$, given that $X_{F}=l$, follows a binomial distribution $\operatorname{Pr}\left[Y_{F}=k \mid X_{F}=l\right]=B_{p}(l, k)=\left(\begin{array}{l}l \\ k\end{array}\right) p^{k}(1-p)^{l-k}$. By the conditional probability formula,

$$
\operatorname{Pr}\left[X_{F}=x \mid Y_{F}=y\right]=\frac{\operatorname{Pr}\left[Y_{F}=y \mid X_{F}=x\right] \operatorname{Pr}\left[X_{F}=x\right]}{\operatorname{Pr}\left[Y_{F}=y\right]}
$$

and by the complete probability formula,

$$
\operatorname{Pr}\left[Y_{F}=y\right]=\sum_{i=y}^{\infty} B_{p}(i, y) \operatorname{Pr}\left[X_{F}=i\right]
$$

We know that flow length distributions have the property of being heavy-tailed. Pareto distribution is the simplest heavy-tailed distribution; its probability mass function is

$$
\operatorname{Pr}\left[X_{F}=x\right]=\beta \alpha^{\beta} / x^{\beta+1}, \alpha, \beta>0, x \geq \alpha
$$

where $\beta$ is called Pareto parameter. Hence Equation (2) can be written as:

$$
\operatorname{Pr}\left[Y_{F}=y\right]=\sum_{i=y}^{\infty} B_{p}(i, y) \beta \alpha^{\beta} / i^{\beta+1}, y \geq \alpha
$$

Lemma 1. Under the assumption that original flow lengths satisfy Pareto distribution, the probability that a sampled flow of length $y(\geq \alpha)$ is sampled from an original flow of length $x$ is

$$
\operatorname{Pr}\left[X_{F}=x \mid Y_{F}=y\right]=\frac{B_{p}(x, y) / x^{\beta+1}}{\sum_{i=y}^{\infty} B_{p}(i, y) \beta \alpha^{\beta} / i^{\beta+1}} .
$$


To describe the properties of the above probability, apply different values of $p$ and $\beta$ to calculate the probability of Lemma 1 . And for fixed $p$ and $\beta$, for each $y(\geq \alpha)$, we find $x$ such that the above probability $\operatorname{Pr}\left[X_{F}=x \mid Y_{F}=y\right]$ is maximized. We have

Lemma 2. Under the assumption of Lemma 1, for fixed $p=1 / N, \beta$ and $y(\geq \alpha)$, the probability $\operatorname{Pr}\left[X_{F}=x \mid Y_{F}=y\right]$ is maximized at $x=N y-n(p, \beta)$. It is increasing as $x$ increases for $x<N y-n(p, \beta)$ and decreasing as $x$ increases for $x>N y-n(p, \beta)$.

Here $n(p, \beta)$ is a binary function with variables $p$ and $\beta$ whose value domain is integer set. Function $n(p, \beta)$ has the following properties:

1) It is a monotone decreasing function on variable $p$, that is , for fixed $\beta$, is decreasing as $p$ increases.

2 ) It is a monotone increasing function on variable $\beta$, that is , for fixed $p$, is increasing as $\beta$ increases.

For example, $n(0.1,0.5)=14, n(0.1,1.0)=18, n(0.1,1.5)=23$. In the concerned network, the length distributions of flows collected in any time interval do not satisfy Pareto distributions with fixed parameter strictly, but they can follow a Pareto distribution with parameter in interval $[0.5,1.5]$ approximately. The value 1.0 is the middle value of interval $[0.5,1.5]$ exactly. Therefore, to compute the conditional probability we assume that original flow length has a Pareto distribution with parameter 1.0 a priori distribution.

\section{$3 \quad$ Estimation Method of Flow Length Distributions}

\subsection{Flow Classification: Large Flow and Small Flow}

Let $g=\left\{g_{j}: j=1,2, \cdots, n\right\}$, where $g_{j}$ is sampled flow frequencies of length $j$, be a set of sampled flow length frequencies, $f=\left\{f_{i}: i=1,2, \cdots, n, \cdots\right\}$ a set of estimated original flow length frequencies. Consider sampling the packets of an original flow of length $N j$ independently with probability $1 / N$, the probability that no packet is sampled is $(1-1 / N)^{N j}=\left((1-1 / N)^{N}\right)^{j} \cdot\left\{(1-1 / N)^{N}\right\}$ is increasing in $N$ and $\lim _{N \rightarrow \infty}(1-1 / N)^{N}=1 / e<0.37$. Thus for a given error $\varepsilon$, we require $(1-1 / N)^{N j}<(1 / e)^{j}<\varepsilon$ and choose $j_{\text {bord }} \geq \max (j(\varepsilon)=\lceil\log (1 / \varepsilon)\rceil, \alpha)$. For example, $j(0.01)=5, j(0.001)=7$. We classify two types of flows based on their probability that no packet is sampled. A flow is labeled as small (S) when it's probability that no packet is sampled is more than $\varepsilon$ and as large $(\mathrm{L})$ when it's probability that no packet is sampled is less than or equal to $\varepsilon$.

\subsection{Estimation for Large Flow}

For a sampled flow of length $j>j_{\text {bord }}$, by Lemma 2 , the original flow length values of the $2 N$ relatively large probabilities are $N(j-1)-n(p, \beta)+1, \cdots, N(j+$ $1)-n(p, \beta)$ where $\beta=1.0$. We estimate the sampled flow is sampled from one 
of the $2 N$ original flows. Then there are $\frac{g_{j}}{2 N}$ sampled flows that are sampled from one of original flows of the above lengths in $g_{j}\left(j>j_{\text {bord }}\right)$ sampled flows. Therefore, for all large flows of length $i>N j_{b o r d}$, we have

$$
f_{i}=\frac{1}{2 N}\left(g_{j}+g_{j+1}\right), \text { where } j=\lfloor(i+n(p, \beta)-1) / N\rfloor .
$$

\subsection{Likelihood Function of Small Flows}

For all small flows of length $i \leq N j_{\text {bord }}$, we estimate as follows:

$$
g_{j}=\sum_{i=j}^{m} B_{p}(i, j) f_{i}
$$

where $m=\max \left\{i: f_{i} \neq 0\right\}$. For $i>N j_{\text {bord }}$, substituting (4) into Equation (5):

$$
\bar{g}_{j}=g_{j}-\sum_{i=N j_{\text {bord }}+1}^{m} B_{p}(i, j) f_{i}=\sum_{i=j}^{N j_{\text {bord }}} B_{p}(i, j) f_{i}, j=l, \cdots, N j_{\text {bord }} .
$$

Because some solutions of Equations (6) may be negative, we don't solve the equations directly. We construct MLE and employ EM algorithm to compute the solutions of Equations (6). For the above some $\bar{g}_{j} \leq 0$, we replace it with $\delta \bar{g}_{i-1}, 0<\delta<1$. Below we only consider all small flows of length $1, \cdots, N j_{\text {bord }}$. Let $\gamma=\sum_{i=1}^{i=N j_{\text {bord }}} \bar{g}_{i}$, and let $\phi_{i}$ denote the frequencies of original flows of length $i$ conditional on at least one of its packets being selected. Our aim is to estimate $\phi=\left\{\phi_{i}\right\}, i=1, \cdots, N j_{b o r d}$ and $\sum_{i} \phi_{i}=1$, from the frequencies $\left\{\bar{g}_{i}\right\}$. We now derive an expression for log-likelihood $L(\phi)$ to obtain $\bar{g}_{i}$ given $\phi$. Here, $c_{i j}=B_{p}(i, j) /\left(1-B_{p}(i, 0)\right)$ is the probability that packets are sampled from a flow of length $i$,conditional on $j \geq 1$, i.e., that the flow is sampled. For any $j$, the function is $\left(\sum_{i=j} \phi_{i} c_{i j}\right)^{\bar{g}_{j}}$. Hence we obtain the likelihood function $\prod_{j=1}^{N j_{\text {bord }}}\left(\sum_{i \geq j} \phi_{i} c_{i j}\right)^{\bar{g}_{j}}$. Therefore the logarithm of likelihood function is

$$
L(\phi)=\prod_{j=1}^{N j_{\text {bord }}} \bar{g}_{j} \log \sum_{i \geq j} \phi_{i} c_{i j}
$$

where $c_{i j}=B_{p}(i, j) /\left(1-B_{p}(i, 0)\right)$. We wish to maximize $L(\phi)$ subject to the constraints $\phi \in \Delta=\left\{\phi: \phi_{i} \geq 0, \sum_{i} \phi_{i}=1\right\}$.

\subsection{EM Algorithm of Small Flows}

Now we adopt a standard iterative approach: the Expectation Maximization (EM) algorithm [9], the standard form is as follows.

Starting with an initial value $\phi^{(0)}$, for example, $\phi^{(0)}=\left\{\frac{\bar{g}_{i}}{\gamma}\right\}$, the algorithm finds $\sup \{L(\phi): \phi \in \Delta\}$, by iterating between the following two steps $(k=$ $0,1, \cdots)$ : 
E step. Let $f_{i j}$ denote the frequencies of original flows of length $i$ from which $j$ packets are sampled. Thus $\bar{g}_{j}=\sum_{i} f_{i j}$, while $\bar{f}_{i}=\sum_{j} f_{i j}$ is the frequency of original flows of length $i$ at least one of whose packets is sampled. Form the complete data likelihood function assuming known $f_{i j}$

$$
L_{c}(\phi)=\sum_{i \geq j \geq 1}^{N j_{\text {bord }}} f_{i j} \log \phi_{i} c_{i j}
$$

Form the expectation $Q\left(\phi, \phi^{(k)}\right)$ of $L_{c}(\phi)$ conditional on the known frequencies $\bar{g}_{j}$, according to a distribution $\phi^{(k)}$ :

$$
Q\left(\phi, \phi^{(k)}\right)=\sum_{i \geq j \geq 1}^{N j_{\text {bord }}} E_{\phi^{(k)}}\left[f_{i j} \mid \bar{g}\right] \log \phi_{i} c_{i j}
$$

M step. Define $\phi^{(k+1)}=\operatorname{argmax}_{\phi \in \Delta} Q\left(\phi, \phi^{(k)}\right)$. From the Legendre equations in the maximization of $Q\left(\phi, \phi^{(k)}\right)$ we have: $\phi_{i}^{(k+1)}=\frac{E_{\phi}(k)\left[f_{i j} \mid \bar{g}\right]}{\gamma}$. Through direct computation of the above conditional expectation we obtain:

$$
\phi_{i}^{(k+1)}=\frac{1}{\gamma} \sum_{i \geq j \geq 1} \frac{\phi_{i}^{(k)} c_{i j} \bar{g}_{j}}{\sum_{l \geq j} \phi_{l}^{(k)} c_{l j}}
$$

Iterate steps E and M until some termination criterion is satisfied. Let $\bar{\phi}$ denote the termination point. We write our estimation of original small flows as $f_{i}=$ $\bar{\phi}_{i} \gamma /\left(1-B_{p}(i, 0)\right), i=1, \cdots, N j_{\text {bord }}$.

\section{Evaluations and Comparison}

Computational complexity. Let $j_{\max }$ denote the maximum sampled flow length. The computation for binomial coefficients of Equations (6) is $O\left(N N j_{\text {bord }} j_{\text {max }}\right)$. Tabulation of the binomial coefficients for the iteration is $O\left(\left(N j_{b o r d}\right)^{2}\right)$. Then for a fixed $\phi_{i}$, each EM iteration is $O\left(\left(N j_{b o r d}\right)^{2}\right)$. For all $\phi_{i}$, completing an EM iteration is $O\left(\left(N j_{\text {bord }}\right)^{3}\right)$. We compare the computational complexity of our method against the best known EM algorithm in [8] for estimating flow distribution from sampled traffic. In [8] for all $\phi_{i}$ completing an EM iteration is $O\left(i_{\text {max }}^{2} j_{\text {size }}\right)$. We collect all IP packet heads during a period of 300 minutes at Jiangsu provincial network border of China Education and Research Network (CERNET) (1Gbps) to do offline experiment. For IP header data during a period of 1 minute, sampling packets with $p=1 / 10$, in our method let $\varepsilon=0.01$, then $j_{\text {bord }}=5$, thus $\left(N j_{\text {bord }}\right)^{3}=50^{3}$. However, $i_{\max }=2000, j_{\text {size }}=200$ in EM algorithm of [8], $i_{\max }^{2} j_{\text {size }}=6400 * 50^{3}$.

Estimation accuracy: We adopt Weighted Mean Relative Difference (WMRD) as our evaluation metric. Suppose the number of original flows of length $i$ is $n_{i}$ and our estimation of this number is $\hat{n}_{i}$. The value of WMRD is given by: $\mathrm{WMRD}=\frac{\sum_{i}\left|n_{i}-\hat{n}_{i}\right|}{\sum_{i}\left(\frac{n_{i}+\hat{n}_{i}}{2}\right)}$. 


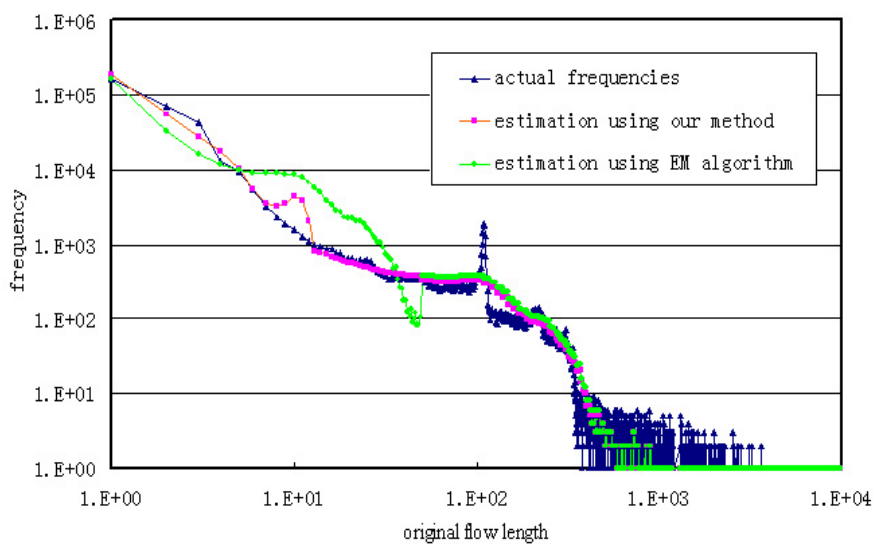

Fig. 1. Comparison of our method and EM algorithm at sampling period $N=10$ for Jiangsu trace

We use three traces in our comparison experiments. The first trace is the first publicly available 10 Gigabit Internet backbone packet header trace from NLANR: Abilence III data set [10]. In our experiments, we used a minute of traffic from the trace. The second trace, which contains packets during a 5 -minute period, was collected at Jiangsu provincial network border of China Education and Research Network (CERNET) on April 17, 2004. The backbone capacity

Table 1. WMRD of our method and EM algorithm

\begin{tabular}{|c|c|c|c|}
\hline trace & Sampling period & WMRD of our method & WMRD of EM algorithm \\
\hline Abilence III & 10 & $17 \%$ & $18 \%$ \\
& 30 & $23 \%$ & $24 \%$ \\
& 100 & $34 \%$ & $37 \%$ \\
\hline Jiangsu & 10 & $20 \%$ & $28 \%$ \\
& 30 & $15 \%$ & $29 \%$ \\
& 100 & $30 \%$ & $39 \%$ \\
\hline Abilence I & 10 & $15 \%$ & $14 \%$ \\
& 30 & $21 \%$ & $23 \%$ \\
& 100 & $31 \%$ & $35 \%$ \\
\hline
\end{tabular}

is $1000 \mathrm{Mbps}$; mean traffic per day is $587 \mathrm{Mbps}$. We call this trace as Jiangsu trace. The third trace, which contains packets during a 10 minute period, was obtained from NLANR: Abilence I [11]. Figure 1 compares the two estimators of Jiangsu trace derived by our method and EM algorithm of [8] at sampling period $N=10$. Observe that they are so close. Table 1 shows the estimation accuracy of our algorithm is close enough to that of EM algorithm. In most cases, our algorithm is much more accurate. 


\section{Conclusions}

Estimating the distribution of flow length is important in a number of network applications. In this paper we present a novel method for estimation of flow length distributions from sampled flow statistics. The main advantage is that it could significantly reduce the computational complexity. The theoretical analysis shows that the computational complexity of our method is well under control. The experimental results demonstrate that our method achieves an accurate estimation for flow distribution.

\section{Acknowledgement}

This work is supported in part by the National Grand Fundamental Research 973 Program of China under Grant No.2003CB314804; the National High Technology Research and Development Program of China (2005AA103001); the Key Project of Chinese Ministry of Education under Grant No.105084; the Jiangsu Provincial Key Laboratory of Computer Network Technology No. BM2003201; Jiangsu Planned Projects for Postdoctoral Research Funds.

\section{References}

1. Duffield, N.G., Lund, C. , Thorup, M.: Charging from sampled network usage. ACM SIGCOMM Internet Measurement Workshop 2001, 245-256, November 2001.

2. Duffield, N.G., Lund, C. , Thorup, M.: Properties and Prediction of Flow Statistics from Sampled Packet Streams. ACM SIGCOMM Internet Measurement Workshop 2002,159-171, November 2002.

3. Feldmann, A. , Caceres, R., Douglis, F., Glass, G., Rabinovich, M.: Performance of Web Proxy Caching in Heterogeneous Bandwidth Environments.IEEE INFOCOM 99,107-116,March 1999.

4. Feldmann, A., Rexford, J., and Caceres, R.: Efficient Policies for Carrying Web Traffic over Flow-Switched Networks. IEEE/ACM Transactions on Networking, 6(1998)673-685.

5. Abhishek Kumar, Jun $\mathrm{Xu}, \mathrm{Li} \mathrm{Li}$, and Jia Wang: Space Code Bloom Filter for Efficient Traffic Flow Measurement.IEEE INFOCOM $2004,1762-1773$.

6. Abhishek Kumar, Minho Sung, Jun (Jim) Xu and Jia Wang: Data streaming algorithms for efficient and accurate estimation of flow size distribution, ACM SIGMETRICS 2004,177-188.

7. Nicolas Hohn, Darryl Veitch: Inverting Sampled Traffic. Internet Measurement Conference 2003. October 27-29, Miami Beach ,Florida, USA. 222-233.

8. Duffield, N.G., Lund, C. , Thorup, M.: Estimating Flow Distributions from Sampled Flow Statistics. IEEE/ACM Transation on Networking, 13(2005) 933-945.

9. Mao shisong, Wang jinglong, and $\mathrm{Pu}$ xiaolong: Advanced Mathematical Statistics. China Higher Education Press, Beijing,1998.

10. NLANR: Abilene-III data set, hppt://pma.nlanr.net/Special/ipls3.html.

11. NLANR:Abilene-I data set, http://pma.nlanr.net/Traces/long/bell1.html. 